\title{
Looking for Young-onset Colorectal Cancer - It is Coming to Asia
}

\author{
Sunny $\mathrm{H}$ Wong ${ }^{1} M D$, PhD, Joseph JY $\underline{\text { Sung }}{ }^{1} M D$, PhD
}

Colorectal cancer $(\mathrm{CRC})$ is the third most common cancer worldwide. In Asia, its incidence is rapidly increasing and has soared to become the top cancer in some countries. ${ }^{1}$ It is currently the top killer in Singapore, affecting more than 1,200 patients each year. While the majority of the cancers are diagnosed in the elderly, recent studies have shown its increasing incidence among young adults aged 50 or below. ${ }^{2,3}$ The recent death of Chadwick Boseman has epitomised this disturbing trend with young-onset $\mathrm{CRC}$, as the disease took the actor's life at the pinnacle of his career. These young-onset CRC patients have implicated a significant health burden to the population due to the cancer mortality, long-term disease morbidity, loss in quality of life, treatment cost and reduced work capability.

In this issue of the Annals, Goh and her colleagues have studied the epidemiology of young-onset CRC in Singapore. ${ }^{2}$ Using a retrospective cohort design, the authors reviewed 99 young patients with sporadic CRC diagnosed between 2010 and 2017. Young cancer patients aged 50 or below at the time of diagnosis were included. Accounting for $\leq 10 \%$ of all CRC, the majority of the patients were male and had distal tumours. Many of these patients had advanced cancers at presentation $(62.6 \%$ in Stages III/IV), and nearly one-third (31.6\%) had developed complications (e.g. intestinal obstruction and perforation) to require emergency surgery. The risk factors and clinical outcomes of these patients were studied.

This study highlights the importance of young-onset CRC. In contrast to its declining incidence in older people, recent longitudinal studies have reported increasing incidences of young-onset CRC worldwide, especially in high-income countries. ${ }^{3,4}$ Similar trends have been observed in several Asian countries, ${ }^{3,5,6}$ including China, Japan, India and notably South Korea, where a steep increase in young-onset CRC has been reported. ${ }^{3,5}$ These young-onset cancers are more likely to be located distally in the left-sided colorectum, present with an advanced stage, exhibit a mucinous or signet ring histology, and be poorly differentiated. ${ }^{7}$ Compatible with these findings, Goh et al. have observed an increasing incidence of young-onset CRC in the Singapore Cancer Registry from 2003 to 2017. ${ }^{2}$ Furthermore, this cohort was characterised by distal colonic and rectal tumours, with patients presenting late with advanced stages and unfavourable histology. On multivariate analysis, the cancer stage and presence of signet ring histology were found to be independent predictors of poor patient survival. The overall 5-year survival rate was $82.0 \%$, with a reasonable survival rate of $83.3 \%$ for Stage III patients, but dropping dismally to $45.0 \%$ for Stage IV patients.

More often than not, epidemiology studies like these can inform us of the disease biology and guide us on health policies. The global increase in the incidence of young-onset $\mathrm{CRC}$, over a relatively short time frame, suggests that environmental factors may have played a major role. It is rather unlikely that genetic changes can solely explain the disease trend in a relatively short time frame. The important environmental factors may include lifestyle changes, such as increased consumption of red and processed meat, reduced fibre intake, alcohol or cigarette consumption, and physical inactivity, which have been associated with the cancer. ${ }^{8}$ Some of these factors are especially relevant to the young population and have site-specific effects on colorectal carcinogenesis, such as processed meat consumption with distal tumours. ${ }^{9}$ Other important factors include obesity, which can promote cancer formation through hyperinsulinemia, systemic inflammation and alternation of the gut microbiota. ${ }^{10}$ A systematic review showed that every $5 \mathrm{~kg} / \mathrm{m}^{2}$ increase in body mass index was associated with an $18 \%$ increased risk of CRC. ${ }^{11}$ Given the rapid dietary transition and remarkable increases in adiposity in the East, ${ }^{12}$ this impact is particularly relevant to the Asian populations. Another related risk factor is early-life antibiotic use, which has been implicated in distal colorectal neoplasia ${ }^{13}$ and hypothesised to account for

${ }^{1}$ Department of Medicine and Therapeutics, the Chinese University of Hong Kong, Hong Kong

Address for Correspondence: Dr Joseph JY Sung, Department of Medicine and Therapeutics, the Chinese University of Hong Kong, Shatin, New Territory, Hong Kong.

Email: jjysung@cuhk.edu.hk 
the steep incidence increase in South Korea. ${ }^{3}$ These early-life antibiotic exposure could disrupt the gut microbiota, alter the metabolic profile and lead to a higher risk of obesity in later life. ${ }^{14}$

Apart from the disease biology, these studies also have important implications on health policies at a population level. As most CRC screening programmes (including that in Singapore) start at the age of 50, these youngonset cancers will be invariably missed. Moreover, as exemplified by the current study by Goh et al., even symptomatic cancer patients would often present late with advanced diseases. This is likely due to the low disease awareness among younger patients, disregarding symptoms or erroneously attributing them to benign conditions, and therefore missing the best window of opportunity to cure the cancer. As such, the American Cancer Society has recommended lowering the age of screening for average risk adults to 45 years of age. ${ }^{15}$ Although the effectiveness of this policy is still debatable and has not been universally adopted, it does highlight the need to continue surveying and revisiting the screening policies with time. With a predilection of tumours in the distal colorectum, the efficacy and costeffectiveness of performing flexible sigmoidoscopy for young patients should represent a sensible idea. Moreover, the use of risk scores to incorporate genetic ${ }^{16}$ and environmental ${ }^{17}$ factors may further help prioritise patients for disease screening, rather than having a one-size-fits-all policy to commence screening at a certain age. Meanwhile, clinicians should remain vigilant with young patients who present with red flag signs of CRC.

The current paper by Goh et al. should be applauded for pioneering young-onset CRC study in Singapore. Nevertheless, it will be worthwhile to expand and study the population-level epidemiology, particularly on its age-standardised incidence, risk factors and clinical outcomes with respect to the older patient group. Given the multi-ethnic population structure and unique socioeconomic background, such data will be exceptionally valuable to help clinicians and policy-makers understand the disease mechanisms and devise mitigating policies for this important cancer.

\section{REFERENCES}

1. $\mathrm{Ng}$ SC, Wong SH. Colorectal cancer screening in Asia. Br Med Bull 2013;105:29-42.
2. Goh SSN, Loo EXL, Lee DJK. Clinical Outcomes in Youngonset Colorectal Cancer Patients. Ann Acad Med Singap 2020;49:848-56

3 Siegel RL, Torre LA, Soerjomataram I, et al. Global patterns and trends in colorectal cancer incidence in young adults. Gut 2019;68:2179-85.

4. Araghi M, Soerjomataram I, Bardot A, et al. Changes in colorectal cancer incidence in seven high-income countries: a populationbased study. Lancet Gastroenterol Hepatol 2019;4:511-8.

5 Sung JJY, Chiu HM, Jung KW, et al. Increasing Trend in YoungOnset Colorectal Cancer in Asia: More Cancers in Men and More Rectal Cancers. Am J Gastroenterol 2019;114:322-9.

6 Lui RN, Choi KKF, Ho JMW, et al. Global Increasing Incidence of Young-Onset Colorectal Cancer Across 5 Continents: A Joinpoint Regression Analysis of 1,922,167 Cases. Cancer epidemiology, biomarkers \& prevention: a publication of the American Association for Cancer Research, cosponsored by the American Society of Preventive Oncology 2019;28:1275-82.

7 Chang DT, Pai RK, Rybicki LA, et al. Clinicopathologic and molecular features of sporadic early-onset colorectal adenocarcinoma: an adenocarcinoma with frequent signet ring cell differentiation, rectal and sigmoid involvement, and adverse morphologic features. Mod Pathol 2012;25:1128-39.

8 Huxley RR, Ansary-Moghaddam A, Clifton P, et al. The impact of dietary and lifestyle risk factors on risk of colorectal cancer: a quantitative overview of the epidemiological evidence. Int J Cancer 2009;125i:171-80.

9 Chao A, Thun MJ, Connell CJ, et al. Meat consumption and risk of colorectal cancer. JAMA 2005;293:172-82.

10 Wong SH, Yu J. Gut microbiota in colorectal cancer: mechanisms of action and clinical applications. Nat Rev Gastroenterol Hepatol 2019;16:690-704.

11 Ning Y, Wang L, Giovannucci EL. A quantitative analysis of body mass index and colorectal cancer: findings from 56 observational studies. Obes Rev 11:12010;9-30.

12 Sung H, Siegel RL, Torre LA, et al. Global patterns in excess body weight and the associated cancer burden. CA Cancer J Clin 2019;69:88-112.

13 Cao Y, Wu K, Mehta R, et al. Long-term use of antibiotics and risk of colorectal adenoma. Gut 2018;67:672-8.

14 Chen LW, $\mathrm{Xu} \mathrm{J}$, Soh SE, et al. Implication of gut microbiota in the association between infant antibiotic exposure and childhood obesity and adiposity accumulation. Int J Obes (Lond) 2020; 44:1508-20.

15 Wolf AMD, Fontham ETH, Church TR, et al. Colorectal cancer screening for average-risk adults: 2018 guideline update from the American Cancer Society. CA Cancer J Clin 2018;68:250-81.

16 Weigl K, Thomsen H, Balavarca Y, et al. Genetic Risk Score Is Associated With Prevalence of Advanced Neoplasms in a Colorectal Cancer Screening Population. Gastroenterology 2018;155:88-98.

17 Sung JJY, Ng SC, Chan FKL, et al. An updated Asia Pacific Consensus Recommendations on colorectal cancer screening. Gut 2015;64:121-32. 